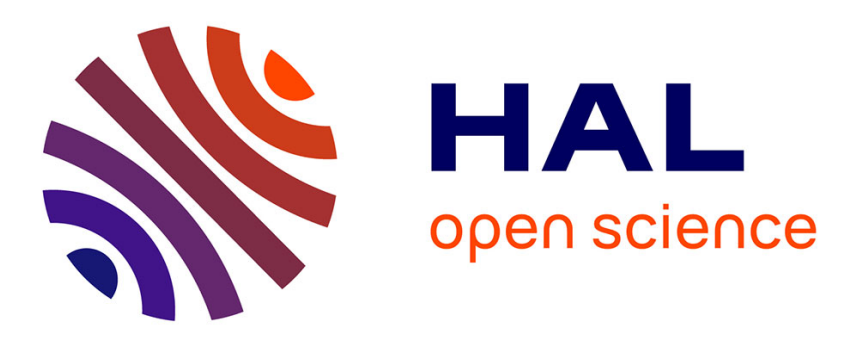

\title{
Low-order modelling of the wake dynamics of an Ahmed body
}

Bérengère Podvin, Stéphanie Pellerin, Yann Fraigneau, Guillaume Bonnavion, Olivier Cadot

\section{- To cite this version:}

Bérengère Podvin, Stéphanie Pellerin, Yann Fraigneau, Guillaume Bonnavion, Olivier Cadot. Loworder modelling of the wake dynamics of an Ahmed body. Journal of Fluid Mechanics, 2021, 927, pp.R6. 10.1017/jfm.2021.788 . hal-03364007

\section{HAL Id: hal-03364007 https://hal.science/hal-03364007}

Submitted on 4 Oct 2021

HAL is a multi-disciplinary open access archive for the deposit and dissemination of scientific research documents, whether they are published or not. The documents may come from teaching and research institutions in France or abroad, or from public or private research centers.
L'archive ouverte pluridisciplinaire HAL, est destinée au dépôt et à la diffusion de documents scientifiques de niveau recherche, publiés ou non, émanant des établissements d'enseignement et de recherche français ou étrangers, des laboratoires publics ou privés. 


\title{
Low-order modelling of the wake dynamics of an Ahmed body
}

\author{
Bérengère Podvin, Stéphanie Pellerin \\ Yann Fraigneau, Guillaume Bonnavion and Olivier Cadot
}

(Received xx; revised xx; accepted xx)

We investigate the large-scale signature of the random switches between two mirrored turbulent wake states of flat-backed bodies. A direct numerical simulation of the flow around an Ahmed body at a Reynolds number of 10000 is considered. Using Proper Orthogonal Decomposition, we identify the most energetic modes of the velocity field and build a low-dimensional model based on the first six fluctuating velocity modes capturing the characteristics of the flow dynamics during and in-between switches. In the absence of noise, the model produces random switches with characteristic time scales in agreement with the simulation and experiments. This chaotic model suggests that random switches are triggered by the increase of the vortex shedding activity. However, the addition of noise results in a better agreement in the temporal spectra of the coefficients between the model and the simulation.

\section{Introduction}

The flat-backed Ahmed geometry (Ahmed et al. 1984) is a useful model to study the complex afterbody aerodynamics and the related base suction of trucks, SUVs and other blunt base vehicles responsible of high drag (Choi et al. 2014). The massive turbulent separation of the flat-backed Ahmed body is dominated by different motions associated with specific time scales (Grandemange et al. 2013; Volpe et al. 2015; Haffner et al. 2020) - which will be expressed in the remainder of the paper in convective time units based on the fluid velocity and body height. First of all, the recirculation zone behind the body is generally instantaneously asymmetric and characterized by switches between the asymmetric quasi-stationary states. The duration of the switches is $O(50)$ convective time units and the mean time between switches is $O(1000)$ convective units. A second type of motion corresponds to vortex shedding in the wake, which can be split into lateral and vertical components and is characterized by nondimensional periods of $O(5)$ convective units. A third type of motion can be associated with the pumping motion of the recirculation zone over time scales of $O(10-20)$ units. These results have been confirmed using different techniques to analyse the flow such as direct Fourier analysis on the velocity field or using proper orthogonal decomposition (POD) by identifying the combinations of the POD modes associated with these global dynamics.

Pavia et al. (2020) made use of the filtering properties of the POD analysis to characterize experimentally the three-dimensional topology of the recirculation and succeed to observe its evolution during the switches. These results were confirmed from the numerical simulation of Fan et al. (2020). These three-dimensional characterizations completed the previous experimental two-dimensional wake measurements of Pavia et al. (2018); Perry et al. (2016). Podvin et al. (2020) recently took advantage of the three-dimensional POD to correlate the different known global dynamics to the base suction (or base drag). They found that the larger the quasi-steady deviation of the wake, the larger the base drag, while large vortex shedding intensity was correlated to lower quasi-steady deviation and 
then to lower drag. Although their simulation reported the permament quasi-steady wake deviation (the reflectional symmetry-breaking state of Grandemange et al. (2013)), it was not long enough to observe wake switching towards the opposite deviation. Recently, the experiment of Haffner et al. (2020) has carefully examined wake switching in relation to base drag and concluded to a base drag reduction of $8 \%$ during the switch, a similar figure found by Grandemange et al. (2014) using a passive control cylinder to symmetrize the wake or previous measurements of base drag due to Evrard et al. (2016) during a switch.

The first numerical wake switching was observed by Dalla Longa et al. (2019) and found to be associated with a vortex loop shedding, also reported by Fan et al. (2020). In an attempt to understand the triggering mechanism for the switch, Hesse \& Morgans (2021) uses different turbulent modelling and pointed out the role of the separation at the front. Whatever the mechanism is, the very long waiting time between the switches and the associated randomness are consistent with rare events of Poisson statistics (Grandemange et al. 2013; Volpe et al. 2015; Cadot et al. 2020) and can be successfully modelled using a Langevin equation as first introduced by Rigas et al. $(2014,2015)$. The modelling has been adapted to the Ahmed body (Brackston et al. 2016; Evrard et al. 2016; Barros et al. 2017) to simulate the bistable dynamics. For these so-called stochastic models, an additional noise is applied on a nonlinear dynamical system and the probability to change equilibrium states is proportional to $\exp \left(-\frac{\Delta V}{\sigma}\right)$. The noise intensity $\sigma$ and the potential barrier between the two equilibrium state $\Delta V$, are deduced from the experimental signals using different identification methods (Rigas et al. 2014; Boujo \& Noiray 2017). Recently, an alternative low-dimensional modelling based on POD was obtained by Podvin et al. (2020) by projecting the Navier Stokes equations on the most energetic POD modes. However, their POD basis did not contain switching events, as no switching occurred during the simulation. Adding noise to the reduced order model was necessary to produce the wake switching similarly to the stochastic models.

The origin of the noise responsible for these switchings still remains an open question. It has been addressed experimentally in Cadot et al. (2020) who attributed the main role to the fluctuation in the wake and not to the small-scale turbulent intensity of the free stream flow. Together with the numerical simulations observation (Dalla Longa et al. 2019; Fan et al. 2020), there are strong evidences that events such as vortex shedding may trigger the switch and one may wonder if a chaotic process involving the largest scales of the flow could generate the observed bistable dynamics. The flow behavior would be described by a nonlinear system as for stochastic models but it would not require additional noise to produce the bistable dynamics. This idea was explored by Varon et al. (2017) who showed that the low-frequency dynamics of the switch appeared to exhibit weakly chaotic behavior.

The aim of the present paper is to pursue the modelling based on the POD of Podvin et al. (2020) but on a longer simulation that contains wake switchings. It will be shown that the POD basis is completed by a new POD mode associated with a switching event. The modelling leads to spontaneous and random switching through a chaotic dynamics implying directly the periodic vortex shedding. The article is organised as follows: section $\S 2$ contains a description of the numerical simulation along with a review of POD. The velocity POD modes are presented in section $\S 3$, then the model is elaborated and discussed in section $\S 4$ and the paper is concluded in section $\S 5$. 
(a)

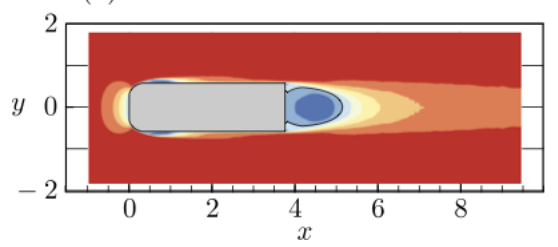

(b)
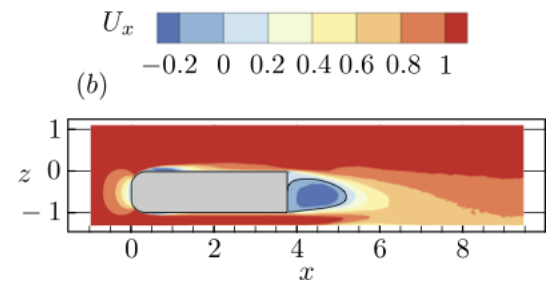

FiguRE 1. Mean velocity (a) mid-height horizontal plane; (b) mid-span vertical plane - The black lines correspond to the isovalue 0 .

\section{Methodology}

The characteristics of the simulation are the same as in Podvin et al. (2020). The dimensions of the squareback Ahmed body are $L=1.124 \mathrm{~m}, H=0.297 \mathrm{~m}, W=$ $0.350 \mathrm{~m}$. The ground height is taken equal to $0.334 H$ in the simulation. The code SUNFLUIDh, which is a finite volume solver, is used to solve the incompressible NavierStokes equations. The Reynolds number based on the fluid viscosity $\nu$, incoming velocity $U$ and Ahmed body height $H$ is $10^{4}$. The temporal discretization is based on a secondorder backward Euler scheme, with implicit treatment of the diffusion terms and explicit representation of the convective terms (Adams-Bashforth scheme). We use $(512 \times 256$ $\times 256$ ) grid points in, respectively, the longitudinal direction $\mathrm{x}$, the spanwise direction $\mathrm{y}$, and the vertical direction $\mathrm{z}$.

The mesh was refined near the body surface and the wall in order to capture the dissipative scales (following Howard \& Pourquie (2002); Fares (2006); Minguez et al. (2008)). In the boundary layers, the cell sizes in the wall-normal and transverse directions ranged from $\eta_{b l}$ to $8 \eta_{b l}$, where the viscous boundary layer thickness $\eta_{b l} \approx 6.71 R e^{-0.9}$ is the smallest wall scale in the simulation. The cell size in the downstream direction ranged from $5 \eta_{b l}$ and $15 \eta_{b l}$. In the wake region, within a downstream distance of $2 H$, cell sizes varied from $2 \eta_{w}$ to $16 \eta_{w}$ in the transverse directions and from $10 \eta_{w}$ to $20 \eta_{w}$ along the downstream direction, where $\eta_{w} \approx 1.2 R e^{-0.75}$ is the Kolmogorov length scale. The simulation time considered in the paper was about 500 convective time units based on the velocity $U$ and height $H$. All reference units will be based on these two quantities. The time step was set to $\Delta t=510^{-4}$ and the CFL number never exceeded 0.4. During the period considered two switches in the deviation could be observed. Figure 1 represents the mean velocity field in a mid-height $(a)$ and a mid-span plane $(b)$. In all figures the origin of the axes is taken at the top and foremost position of the Ahmed body in the vertical symmetry plane. The longitudinal axis is oriented downstream, the vertical axis pointing in the upward direction and the third component is chosen accordingly to obtain a right-handed coordinate system.

Two switches were observed during the total length of the simulation. We note that in experiments (Evrard et al. 2016) or in simulations (Hesse \& Morgans 2021) it was not unusual to observe inter-switch times of only a few hundred convective time units, which is consistent with the fact that the advent of switches is governed by a Poisson distribution of mean 1000 time units (Grandemange et al. 2013).

The main tool of analysis used in this paper is Proper Orthogonal Decomposition (POD) (Lumley 1967), which we apply to the velocity field $\underline{u}(\underline{x}, t)$ defined on a spatial domain $D$. The field can be expressed as a superposition of spatial modes

$$
\underline{u}(\underline{x}, t)=\sum_{k \geqslant 0} a_{k}^{v}(t) \underline{\phi}_{k}(\underline{x})
$$

where the spatial modes $\underline{\phi}_{k}$ are orthogonal (and can be made orthonormal), i.e 
$\int_{D} \underline{\phi}_{k}(\underline{x}) \cdot \underline{\phi}_{m}(\underline{x}) d \underline{x}=\delta_{k m}$, while the amplitudes $a_{k}$ are uncorrelated. The modes can be ordered by decreasing energy $\lambda_{1} \geqslant \lambda_{2} \geqslant \ldots \geqslant \lambda_{k}=\left\langle\tilde{a}_{k}^{2}\right\rangle$, where $<$. > represents a time average. Since the full velocity field is considered and the database is symmetrized, the mode 0 corresponds to the symmetric part of the time-averaged field (Podvin et al. 2020), which we checked was essentially equal to the time-averaged field (since the coefficient $a_{0}$ is constant within less than $0.3 \%$ ). The amplitudes $a_{k}^{v}$ can be obtained from the knowledge of the spatial modes by projection of the vector field $\underline{u}$ onto the spatial modes

$$
a_{k}^{v}(t)=\int_{\Omega} \underline{u}(\underline{x}, t) \cdot \underline{\phi}(\underline{x}) d \underline{x} .
$$

One can obtain the modes from a set of $N$ snapshots by solving $\bar{C}_{i j} \cdot A_{j k}=\lambda_{k} A_{i k}$, where $A_{j k}=a_{k}\left(t_{j}\right)$ and $\bar{C}$ is the temporal autocorrelation matrix: $\bar{C}_{i j}=\frac{1}{N} \int_{D} \underline{u}\left(\underline{x}, t_{i}\right) \cdot \underline{u}\left(\underline{x}, t_{j}\right) d \underline{x}$ (method of snapshots). When appropriate we will consider normalized amplitudes defined as $a_{k}=a_{k}^{v} / \sqrt{\lambda_{k}}$.

The decomposition was applied to the velocity field, using the symmetrization procedure described in Podvin et al. (2020) in order to guarantee all POD modes to have the same reflectional symmetry $y \rightarrow-y$ as the geometry. Consequently the POD modal basis is made to respect the symmetry of the forcing geometry. Results shown in this paper were based on a set of 1200 snapshots, 600 of which are extracted from the simulation, and 600 are obtained through the reflection symmetry. The original 600 snapshots span 300 convective time units, 200 of which correspond to the duration of the first switch. Comparison with another set of snapshots based on $2 \times 200$ samples acquired spanning the duration of the second switch did not show any significant differences in the shape of the modes.

\section{POD analysis of the velocity field}

The POD modes of the full velocity field are shown in figure $2(a, b)$ as the component $\phi_{k x}$ of the modes $k$. They can be compared with those obtained using a database that does not contain any switching, described in Podvin et al. (2020). The main difference in the decompositions is the emergence of a new mode (mode 2 in figure $2 a, b$ ), that will be called the switch mode (please note that modes are indexed from 0 in the present paper and 1 in Podvin et al. (2020)). The new second mode creates a strong dissymmetry between the upper and the lower part of the near wake. The mode is symmetric and is essentially streamwise invariant in the far wake. The temporal spectra of the amplitudes are also represented for each mode in figure 2(c). Modes 1 and 2 are characterized by low frequencies. We can notice a slight local increase in the spectrum around the nondimensional frequency (or Strouhal number) of 0.2 - we will come back to this point in the next section.

The next four modes (modes 3 to 6 ) correspond to Von Kármán shedding modes in the horizontal and in the vertical direction. Modes 3 and 4 are characterized by a nondimensional frequency of 0.19 and modes 5 and 6 are characterized by a frequency of 0.23 . These four modes also display a slight peak at 0.8 , which can be seen to match the frequency of the Kelvin-Helmholtz vortices observed along the body sides and originating from the separations at the nose in the modes $3,4,5,6$.

It is possible to describe the large-scale dynamics with a relatively low number of modes as evidenced by figure 3 which shows three instantaneous fields at the beginning $(a)$, in the middle $(b)$ and at the end $(c)$ of the first switch. Each time stamp of the switch is defined as $a_{1}^{v}$ maximum, zero and minimum. The times are materialized with red vertical 


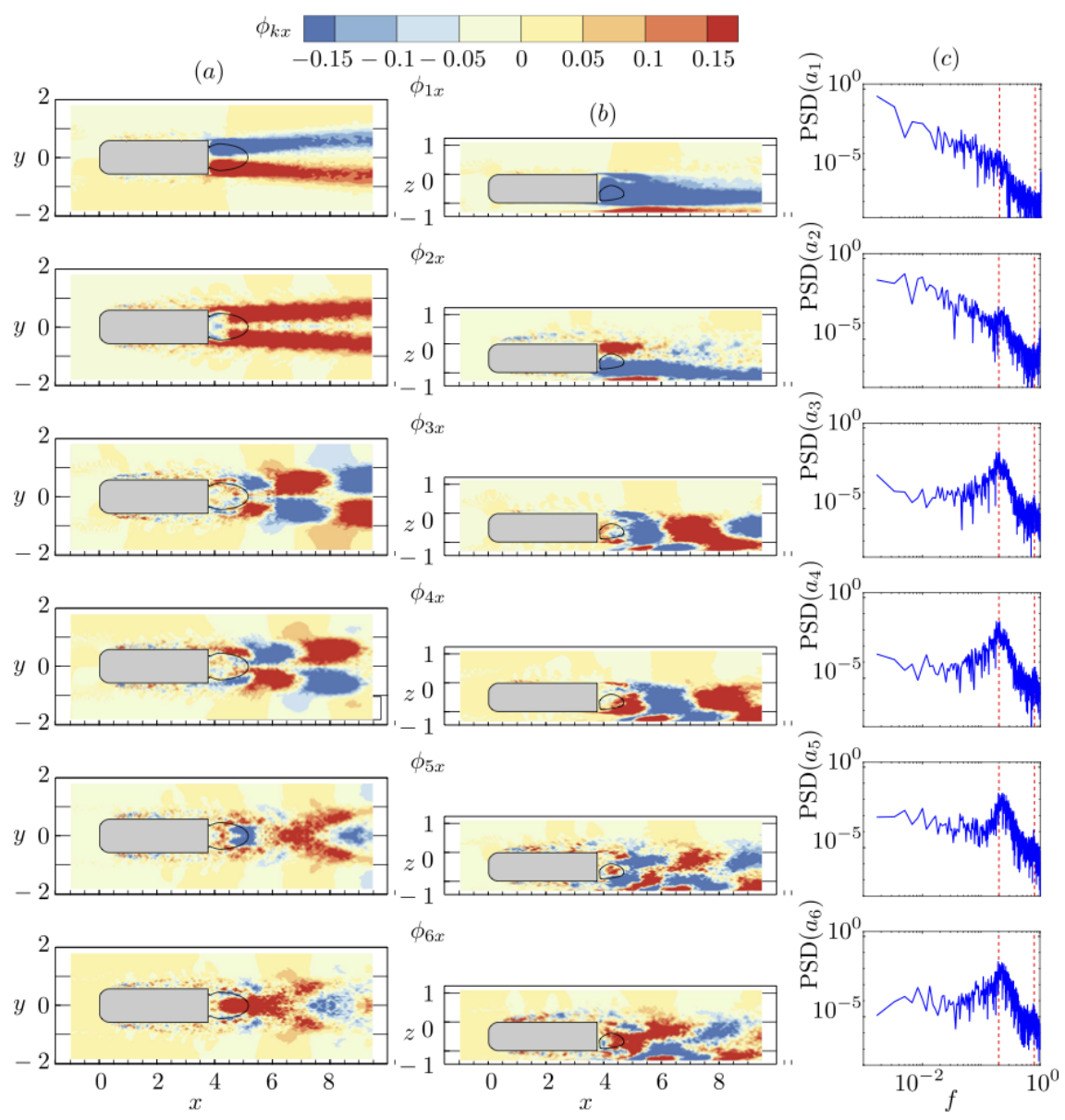

Figure 2. Cross-sections of POD velocity mode streamwise component: $(a)$ on mid-height plane; (b) on mid-span plan for the symmetric modes $k=2,3,4$ and at the position $y=0.4 H$ for the antisymmetric modes $k=1,5,6$. The black line corresponds to the contour $U_{x}=0 ;(c)$ Amplitude temporal spectra - the red dashed lines correspond to the frequencies $f=0.2$ and $f=0.8$.

lines in the shaded area of figure 4. The full fields (left side of figure 3 ) can be compared with their reconstructions (right side of figure 3 ) based on the mean (figure 1) and the 6 most energetic POD modes (figure $2 a, b$ ). The time evolution of the first two fluctuating amplitudes $a_{1}^{v}$ and $a_{2}^{v}$ are represented in figure 4 . We can see that the coefficient of the switch mode $a_{2}^{v}$ is maximum during the switch. In the next section, we examine if the large-scale signature of the switch can be reproduced with a low-dimensional model.

\section{Low-dimensional model}

\subsection{Derivation}

We give a brief review of the derivation process (see also Podvin et al. (2020); Soucasse et al. (2020)). The Navier-Stokes equations are projected onto the basis of the spatial velocity modes $\underline{\phi}_{k}$ for a given truncation $k \in\left[1, \ldots, N_{T}\right]$ and a model, or system of $N_{T}$ ODEs is obtained. The model is of the form

$$
\dot{a}_{k}^{v}=L_{k m} a_{m}^{v}+Q_{k m p} a_{m}^{v} a_{p}^{v}+T_{k} .
$$




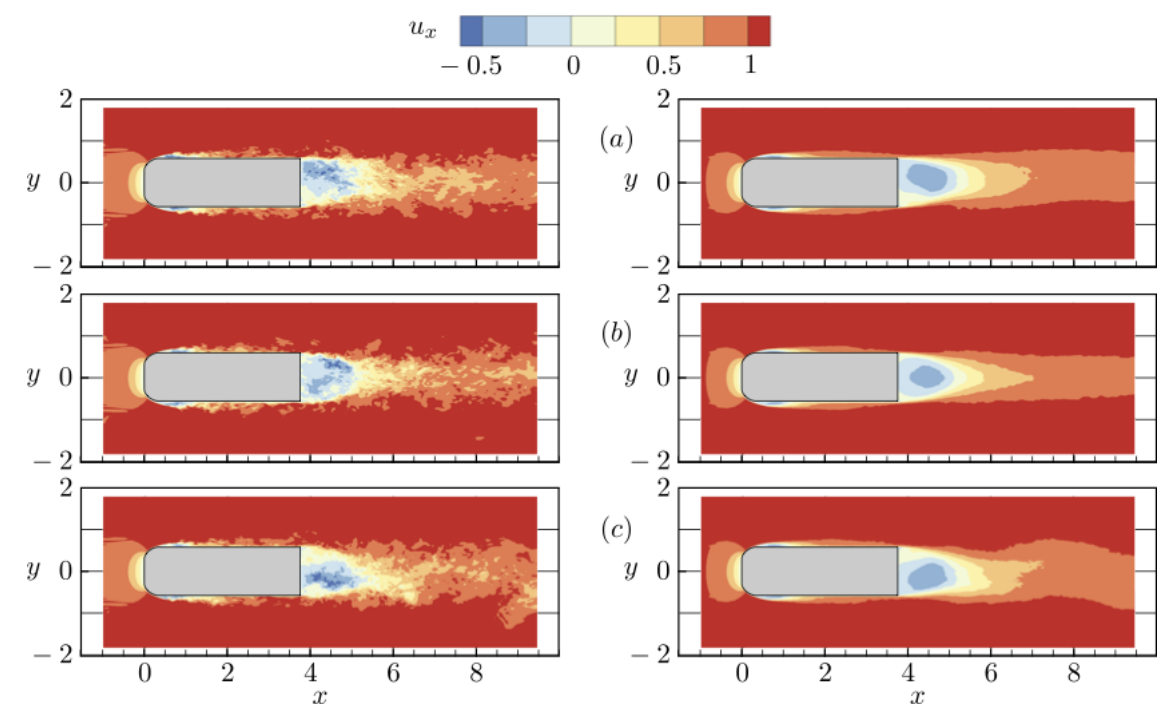

Figure 3. Velocity in a horizontal mid-plane at (a) the beginning, (b) during when $a_{1}^{v}=0$, and (c) at the end of the switch; left) instantaneous field; right) reconstructed field using the mean and the 6 first fluctuating modes.
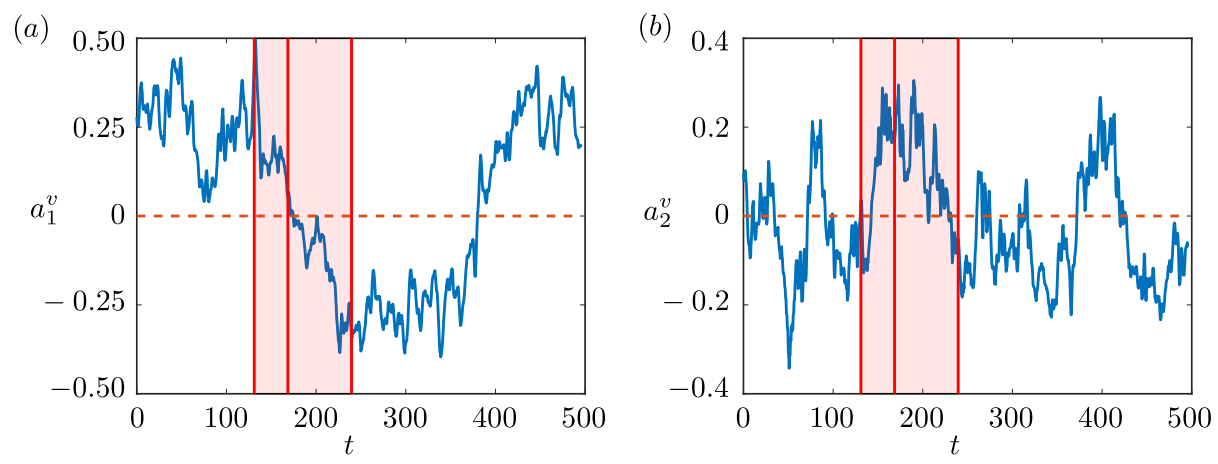

Figure 4. Amplitudes of the POD velocity modes $(a) a_{1}^{v} ;(b) a_{2}^{v}$. The vertical lines in the shaded area corresponding to the switch represent the times of figure 3 .

The linear term $L_{k m}$ is due to the viscous dissipation $L_{k m}=\int \nu \Delta \underline{\phi}_{m} \cdot \underline{\phi}_{k} d \underline{x}$ and is found to be essentially diagonal. The quadratic terms $Q_{k m p}$ can be written in symmetric form as

$$
\left.Q_{k m p}=\frac{1}{2}\left(2-\delta_{m p}\right) \int\left(\underline{\phi}_{p} \cdot \nabla \underline{\phi}_{m}\right)+\underline{\phi}_{m} \cdot \nabla \underline{\phi}_{p}\right) \cdot \underline{\phi}_{k} d \underline{x} .
$$

They represent the nonlinear interaction of the resolved modes, including the mean field corresponding to mode $0 . T_{k}$ is a closure term representing the contribution of the unresolved stresses (associated with the modes excluded from the truncation) to the evolution of the amplitude $a_{k}$. As has been shown in Podvin \& Sergent (2017), the closure term can be modelled as $T_{k}=-\alpha_{k} \sum_{p=1}^{N_{T}}\left(\lambda_{p}+\left|a_{p}^{v}\right|^{2}\right) a_{k}^{v}+\epsilon_{k}$, where $\lambda_{p}$ is the energy of mode $\mathrm{p}$, and $\epsilon_{k}$ is a noise-like perturbation. The value of $\alpha_{k}$ is determined by requiring that for some equilibrium state $a_{p}^{v, e q}$ inferred from the DNS, the total contribution $A_{k}=L_{k k} a_{k}^{v}+Q_{k k 0} a_{k}^{v}+T_{k}$ cancels when $a_{k}^{v}=a_{k}^{v, e q}$ for $k=1, \ldots, 6$, and $\epsilon_{k}=0$, which yields: 


$$
\alpha_{k}=\frac{L_{k k}+Q_{k k 0}}{\sum_{p=1}^{N_{T}}\left(\lambda_{p}+\left|a_{p}^{v, e q}\right|^{2}\right)} \quad \text { (4.3) which yields } A_{k}=\alpha_{k} a_{k}^{v} \sum_{p=1}^{N_{T}}\left(\left|a_{p}^{v, e q}\right|^{2}-\left|a_{p}^{v}\right|^{2}\right) \text {. }
$$

Since equation (4.4) is derived from relatively crude modelling assumptions, some adjustment in the definition of $A_{k}$ may be needed in the model.

\subsection{Seven-mode truncation}

In what follows we consider a seven-mode truncation for the full field $\left(N_{T}=6\right)$. Since the amplitude of the mean field is constant, i.e. $a_{0}=1$, the model is six-dimensional. The model includes the deviation mode, the switch mode and the Von Kármán shedding modes. We note that the model is different from Podvin et al. (2020)'s which did not include the switch mode $a_{2}$. If only the deviation mode is retained in the model, the system is formally analogous to Rigas et al. (2015)'s model. The quadratic interactions coefficients were thresholded: values smaller than $5 \times 10^{-3}$ were not included in the model. It remains to determine the small-scale modelling parameters $\alpha_{k}$ and $\epsilon_{k}$. The noise level rms $\sigma_{k}=<\epsilon_{k}^{2}>^{1 / 2}$ was chosen to be equal to a constant value $\sigma$ for all modes and was estimated to be on the order of $\sigma \sim \alpha_{k} \sum_{p \geqslant 1}^{N_{T}} \lambda_{p} \sim 0.1$. In order to determine $\alpha_{k}$, we use equation (4.3). The equilibrium state was defined as $a_{1}^{e q}= \pm 1, a_{2}^{e q}=0$ (no switch mode), $\left|a_{2 i-1}^{e q}\right|^{2}+\left|a_{2 i}^{e q}\right|^{2}=2$ for $i=2,3$ (constant vortex shedding intensity). Given the eigenvalues $\lambda_{2}=0.057, \lambda_{3}=0.015$ and $\lambda_{i} \sim 0.01$ for $i=3, \ldots, 6$, equation (4.3) yields values of $\alpha_{k}$ in the range [0.85, 1.25]. Since the small scales modelling is relatively crude, we used a single value $\alpha_{k}=\alpha=1$ for all modes. The system can then be written in normalized form as:

$$
\begin{aligned}
& \dot{a}_{0}=0 \\
& \dot{a}_{1}=A_{1}+0.011 a_{1} a_{2}+0.019 a_{0} a_{3}+0.023 a_{0} a_{4}+0.045 a_{3} a_{5}+0.005 a_{1} a_{6}+\epsilon_{1} \\
& \dot{a}_{2}=A_{2}+0.12 a_{0} a_{5}+0.11 a_{0} a_{6}+0.007 a_{2} a_{3}-0.018 a_{1}^{2}+0.018 a_{0}^{2}+\epsilon_{2} \\
& \dot{a}_{3}=A_{3}+1.08 a_{0} a_{4}-0.02 a_{0} a_{1}+0.037 a_{1} a_{6}-0.025 a_{2} a_{4}+\epsilon_{3} \\
& \dot{a}_{4}=A_{4}-1.08 a_{0} a_{3}-0.16 a_{0} a_{1}-0.04 a_{1} a_{5}+0.025 a_{2} a_{3}+\epsilon_{4} \\
& \dot{a}_{5}=A_{5}+1.18 a_{0} a_{6}-0.13 a_{0} a_{2}+0.035 a_{2} a_{3}-0.013 a_{1} a_{3}+0.029 a_{1} a_{4}-0.013 a_{2}^{2} \\
& \dot{a}_{6}=A_{6}-1.18 a_{0} a_{5}-0.26 a_{0} a_{2}-0.026 a_{2} a_{5}-0.02 a_{1} a_{3}+\epsilon_{6}
\end{aligned}
$$

where the numerical quadratic coefficients $\tilde{Q}_{k m p}$ are equal to $\frac{\sqrt{\lambda_{m} \lambda_{p}}}{\lambda_{k}} Q_{k m p}$, and $A_{k}$ is based on the following nondimensional adaptation of (4.4) to account for the energy transfer to the unresolved scales:

$$
A_{k}=\alpha_{k} a_{k} \sum_{p=1}^{N_{T}}\left(\beta_{k}\left|a_{p}^{v, e q}\right|^{2}-\left|a_{p}^{v}\right|^{2}\right)=\alpha a_{k} \sum_{p=1}^{6} \lambda_{p}\left(\beta_{k}\left|a_{p}^{e q}\right|^{2}-\left|a_{p}\right|^{2}\right),
$$

where $\beta_{k}=1$ for $k \leqslant 2$ and $\beta_{k}=0.8$ for $k \geqslant 3$. The mode $a_{0}$ corresponding to the mean velocity is equal to 1 . We point out that the evolution of a mode of a given symmetry is governed by interactions of modes obeying the same symmetry. The deviation mode is therefore controlled by its interaction with the switch mode, but also by cross-interactions between vortex shedding modes of opposite symmetries.

\subsection{Results}

The model was integrated over 20000 convective time units with and without noise. We first consider the integration without noise $(\sigma=0)$. The time series are represented 
(a)
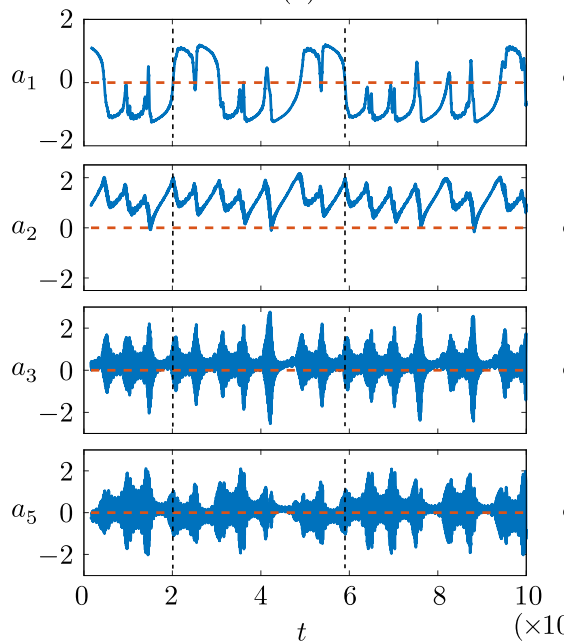

(b)
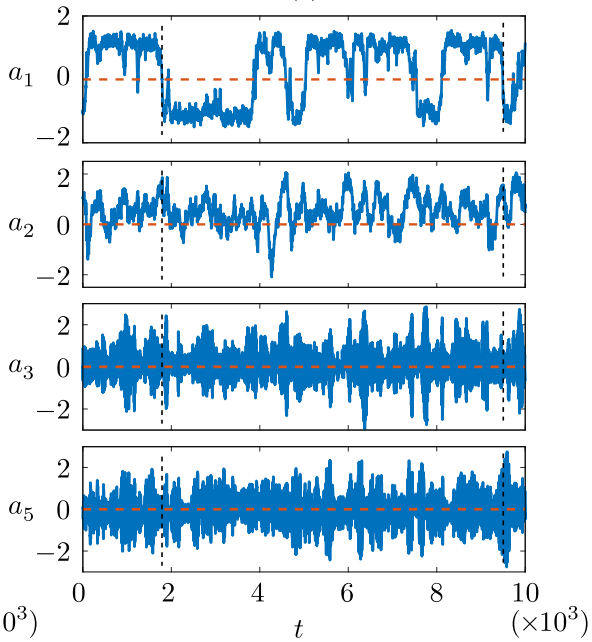

Figure 5. Amplitudes of POD modes 1, 2, 3 and 5 integrated in the model $(a)$ without and (b) with noise. Vertical dashed lines locate time at which $a_{1}=0$ for two switching examples.

in figure 5(a) and can be compared with the normalized amplitudes obtained from the simulation in figure 4 . We can see that the model displays chaotic dynamics characterized by switches. The inter-switch time is random and on the order of a few thousand units, in agreement with Grandemange et al. (2013). The duration of the switch itself is $\mathrm{O}(100)$ convective units, in good agreement with the simulation. The intensity of the vortex shedding increases drastically during the switches (figure $5 a$, bottom rows). This suggests that the shedding modes symmetrize the wake. At the onset of the switch, $a_{2}$ increases then decreases sharply. It becomes strongly positive and reaches its maximum when $a_{1}$ goes through zero, in agreement with the DNS (figure 4). The model therefore does not only display spontaneous switches, but also captures the specific dynamics of the switch mode $a_{2}$. We then consider the integration of the model with noise. Figure $5(b)$ shows the evolution of the amplitudes for a model with a noise rms of $\sigma=0.07$. As expected, the inter-switch time interval decreased with an increasing noise level but remained on the order of $10^{3}$ units for $\sigma \in[0.05,0.1]$. As can be seen in figure $5(b)$, the noise may trigger a switch without any shedding intensity increase as shown for the first switch example (see caption). However, the second switch example is clearly associated with an $a_{5}$ increase corresponding to the shedding in the vertical direction.

The addition of noise leads to time evolutions which are closer to the DNS amplitudes, as can also be seen in figure 6 , which compares the phase portraits in the $\left(a_{1}, a_{2}\right)$ space for the model without noise, the model with noise and the DNS. We note that when noise is added to the model, $a_{2}$ can take negative values in-between switches, which is more consistent with DNS observations (there is still a discrepancy, which is due to the choice of the particular equilibrium $a_{2}^{e q}=0$ ). This is confirmed by comparison of the spectra shown figure 7 . The characteristic frequencies associated with vortex shedding are clearly identified in the model without noise, while an excellent agreement in both the dominant frequencies and in the general shape and levels of the spectra is observed between the model with noise and the DNS. The model therefore captures the main time scales of the dynamics, including those of the switch.

Further insight into the physics of the model can be obtained by artificially manipulating the energy levels of the modes. The results of these numerical experiments are 
(a)

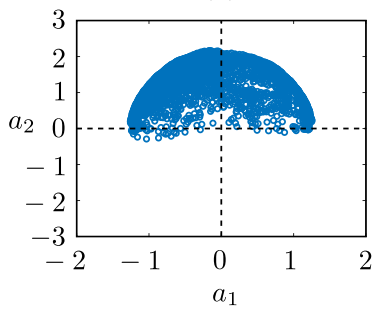

(b)

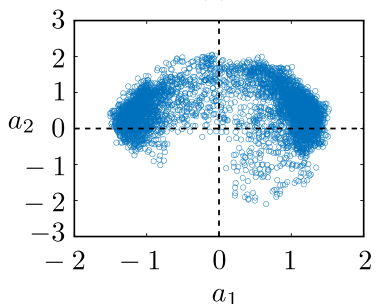

$(c)$

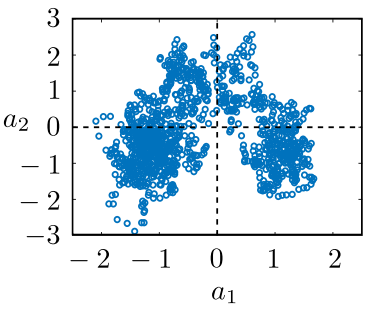

Figure 6. Phase portraits in the $\left(a_{1}, a_{2}\right)$ space: $(a)$ in model without noise; $(b)$ in model with noise level $\sigma=0.07 ;(c)$ in DNS.

(a)
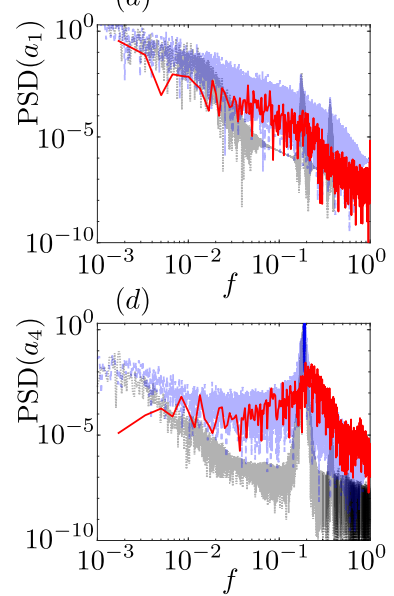

$(b)$
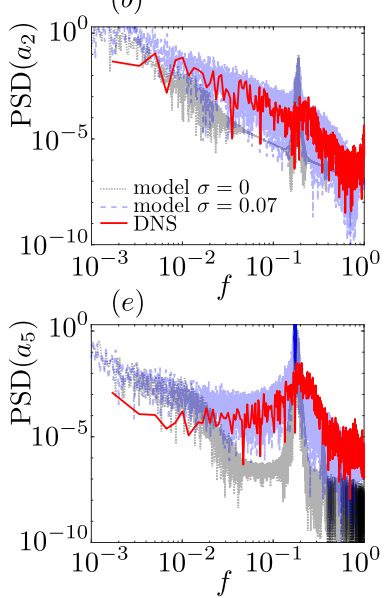

$(c)$

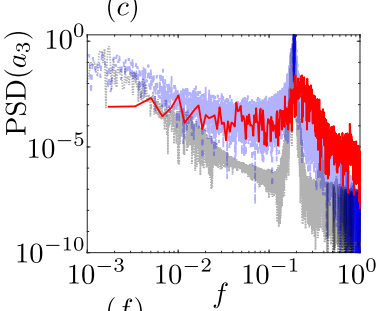

FIGURE 7. Comparison of the spectra of the velocity mode amplitudes $a_{k}$ in the DNS and in the model without and with a noise level $\sigma$.

presented in figure 8. Excluding the switch mode from the model (see figure $8 a$ ) i.e. setting $a_{2}=0$ suppresses switches in the absence of noise - the system remains near one of the equilibrium states - and strongly limits them in the presence of noise which was obtained with the same noise perturbation as in figure $5(b)$. This is confirmed by linear stability of the quasi-steady state, according to which the equilibrium is stable if the switch mode is omitted from the model. The influence of the vortex shedding intensity can be examined by modifying the eigenvalues $\lambda_{i} \rightarrow s \lambda_{i}$ for $i=3, \ldots, 6$. This leads to modifications in the quadratic terms $\tilde{Q}_{k m p}$ as well as in $A_{k}$, with a new equilibrium state $\left|a_{2 i-1}^{e q}\right|^{2}+\left|a_{2 i}^{e q}\right|^{2}=2 s$ for $i=2,3$ and a new value of $\alpha$ in order to satisfy equations $(4.3,4.4)$. Integration of the model without noise is presented in figure $8(b)$ for $s=1.5$ and $s=0.5$. Spontaneous switching is more frequent when the energy is increased by $50 \%(s=1.5)$, as can be seen from comparison with figure 5 , and is suppressed when the vortex shedding energy is reduced to $50 \%(s=0.5)$ of its expected value. This suggests that both the switch and vortex shedding modes are key players in the switching process. We speculate that the vortex shedding modes could be considered as a periodic forcing that introduces chaotic behavior at longer time scales in the 2-D system constituted by the deviation and the switch modes, in a manner that could be reminiscent of forced nonlinear oscillators such as the Duffing or the Van der Pol equation (Guckenheimer \& Holmes 1983). 
(a)

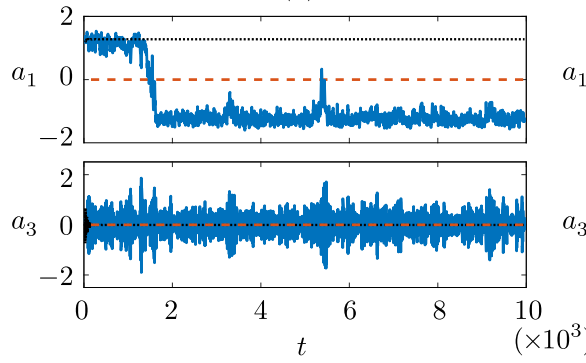

(b)

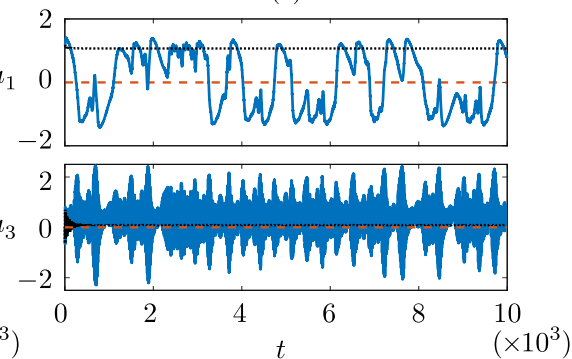

Figure 8. Amplitudes of POD modes 1 and 3 in modified models: $(a)$ models with no switch mode $a_{2}=0$ with (solid line) and without noise (dotted line), $(b)$ models with no noise and modified vortex shedding energy $s \lambda_{i}, i=3, \ldots 6$ (see text) for $s=1.5$ (solid line) and $s=0.5$ (dotted line).

\section{Conclusion}

We have investigated switches of the wake deviation in the flow around an Ahmed body using POD analysis of the velocity field. Along with the deviation and vortex shedding modes that were previously detected in the simulation, a new mode, called the switch mode, was identified. The effect of the mode during the switch is to speed up fluid on the upper part of the wake, and to slow it down on its lower part. A sixdimensional model consisting of the deviation, switch and four modes associated with vortex shedding was educed from the Navier-Stokes equations. In the absence of noise, the model displays chaotic dynamics, with switches occurring intermittently on a time scale of $O\left(10^{3}\right)$ convective units and lasting for about 100 units. The model captures the strong variations of the switch mode, which reaches its maximum when the deviation amplitude is zero, and predicts a sharp increase of the vortex shedding intensity at the onset of the switch. The agreement with the simulation is further strengthened when noise is added to the model, as it results in a very good prediction of the temporal spectra of the large scales. Numerical experiments highlight the importance of both switch and vortex shedding modes in the switching process.

\section{REFERENCES}

Ahmed, S.R., Ramm, G. \& Faitin, G. 1984 Some salient features of the time-averaged ground vehicle wake. SAE Technical Paper Series 840300.

Barros, D., Borée, J., Cadot, O., Spohn, A. \& Noack, B. R. 2017 Forcing symmetry exchanges and flow reversals in turbulent wakes. Journal of Fluid Mechanics $\mathbf{8 2 9}$.

Boujo, Edouard \& Noiray, Nicolas 2017 Robust identification of harmonic oscillator parameters using the adjoint fokker-planck equation. Proceedings of The Royal Society A 473.

Brackston, R.D., Cruz, J.M. Garci De La, Wynn, A., Rigas, G. \& Morrison, J.F. 2016 Stochastic modelling and feedback control of bistability in a turbulent bluff bodywake. $J$. Fluid Mech. 802, 726-749.

Cadot, O., Almarzooqi, M., Legeai, A., Parezanović, V. \& Pastur, L. 2020 On threedimensional bluff body wake symmetry breaking with free-stream turbulence and residual asymmetry. Comptes Rendus. Mécanique 348 (6-7), 509-517.

Choi, H., Lee, J. \& PARk, H. 2014 Aerodynamics of heavy vehicles. Annual Review of Fluid Mechanics 46, 441-468.

Dalla Longa, L., Evstafyeva, O. \& Morgans, A. S. 2019 Simulations of the bi-modal wake past three-dimensional blunt bluff bodies. Journal of Fluid Mechanics 866, 791-809.

Evrard, A., Cadot, O., Herbert, V., Ricot, D., Vigneron, R. \& Delery, J. 2016 Fluid 
force and symmetry breaking modes of a $3 \mathrm{~d}$ bluff body with a base cavity. Journal of Fluids and Structures 61, 99-114.

FAn, Y., ChaO, X., Chu, S., YAng, Z. \& CAdot, O. 2020 Experimental and numerical analysis of the bi-stable turbulent wake of a rectangular flat-backed bluff body. Phys. Fluids 32, 105111.

FAREs, EhAB 2006 Unsteady flow simulation of the ahmed reference body using a lattice boltzmann approach. Computers and Fluids 35 (8), 940-950, proceedings of the First International Conference for Mesoscopic Methods in Engineering and Science.

Grandemange, M., Gohlke, M. \& Cadot, O. 2013 Turbulent wake past a three-dimensional blunt body. part 1. global modes and bi-stability. Journal of Fluid Mechanics 722, 51-84.

Grandemange, M., Gohlke, M. \& Cadot, O. 2014 Turbulent wake past a three-dimensional blunt body. Part 2. Experimental sensitivity analysis. Journal of Fluid Mechanics 752, 439-461.

Guckenheimer, J. \& Holmes, P. 1983 Nonlinear oscillations, dynamical systems and bifurcations of vector fields. Springer-Verlag.

Haffner, Y., Borée, J., Spohn, A. \& Castelain, T. 2020 Mechanics of bluff body drag reduction during transient near-wake reversals. Journal of Fluid Mechanics 894, A14.

Hesse, F. \& Morgans, A.-S. 2021 Simulation of wake bimodality behind squareback bluffbodies using les. Computers \& Fluids 223, 104901.

Howard, R J A \& Pourquie, M 2002 Large eddy simulation of an Ahmed reference model. Journal of Turbulence $\mathbf{3}, \mathrm{N} 12$.

Lumley, J.L. 1967 The structure of inhomogeneous turbulent flows. In Atmospheric Turbulence and Radio Wave Propagation (ed. A.M Iaglom \& V.I Tatarski), pp. 221-227. Nauka, Moscow.

Minguez, M., Pasquetti, R. \& Serre, E. 2008 High-order large-eddy simulation of flow over the "Ahmed body" car model. Physics of Fluids 20, 0951011.

Pavia, G., Passmore, M. \& SArdu, C. 2018 Evolution of the bi-stable wake of a square-back automotive shape. Exp. in Fluids 59, 2742.

Pavia, G., Passmore, M., Varney, M. \& Hodgson, G. 2020 Salient three-dimensional features of the turbulent wake of a simplified square-back vehicle. J. Fluid Mech. 888, A33.

Perry, A.K., Pavia, G. \& Passmore, M. 2016 Influence of short rear end tapers on the wake of a simplified square-back vehicle: wake topology and rear drag. Experiments in Fluids 57 (11).

Podvin, B., Pellerin, S., Fraigneau, Y., Evrard, A. \& Cadot, O. 2020 Proper orthogonal decomposition analysis and modelling of the wake deviation behind a squareback ahmed body. Phys. Rev. Fluids. 6 (5), 064612.

Podvin, B. \& Sergent, A. 2017 Precursor for wind reversal in a square rayleigh-bénard cell. Physical Review E 05 (1), 013112.

Rigas, G., Morgans, A.S, Brackston, R.D. \& Morrison, J.F. 2015 Diffusive dynamics and stochastic models of turbulent axisymmetric wakes. J. Fluid Mech. 778 (R2).

Rigas, G., Oxlade, A.R., Morgans, A.S \& Morrison, J.F. 2014 Low-dimensional dynamics of a turbulent axisymmetric wake. J. Fluid Mech. 755, 159.

Soucasse, L., Podvin, B., Rivière, P. \& Soufiani, A. 2020 Reduced-order modelling of radiative transfer effects on rayleigh-bénard convection in a cubic cell. J. Fluid Mech. 898, A2.

Varon, E., Eulalie, Y., Edwige, S., Gilotte, P. \& Aider, J.L. 2017 Chaotic dynamics of large-scale structures in a turbulent wake. Phys. Review Fluids . 2 (034604).

Volpe, R., Devinant, P. \& Kourta, A. 2015 Experimental characterization of the unsteady natural wake of the full-scale square back ahmed body: flow bi-stability and spectral analysis. Exp. in Fluids 56 (5), 1-22. 